\title{
Research and Deployment on Smart Home System based on Middle- ware Technology and Cross-Platform Communication
}

\author{
Zhipeng $\mathrm{Xu}^{1}$, Zisu Meng ${ }^{2}$, Xiaoxiao Fang ${ }^{3}$, Harry Wang ${ }^{4}$ \\ ${ }^{1}$ Beijing University of Posts and Telecommunications, International School, E-commerce with \\ Laws; \\ ${ }^{2}$ School of Instrument Science and Opto-electronics Engineering, Beihang University; \\ ${ }^{3}$ Wuhan Foreign Languages School, Wuhan, China; \\ ${ }^{4}$ Department of Electrical and Computer Engineering, Cornell, USA.
}

Keywords: Smart Home, Middleware Technology, Cross-Platform, Communication.

\begin{abstract}
With the rapid development of electronic technology, household devices have been developed to automatically sense environmental changes by automatic sensing system. Smart home, the trend of future lifestyle, has been payed specially attention both in academia and industry. Smart home system covers the electronic technology, network technology and control technology based on the network connection with the terminal equipments. These terminal equipments will be related to the household life within the visible interphone, home security, home appliance control, remote monitoring, remote medical diagnosis, nursing system, online education system, home audio system and other subsystems. Our research introduces the middleware technology and cross-platform communication paradigm into classic smart home system to enhance the feasibility, which optimizes the traditional communication protocol and simplifies the deployment procedures.
\end{abstract}

\section{Introduction}

With the constant improvement of science and technology, modern technology promotes the progress of human society and also gradually changes the structure of the pursuit of human beings. From the pursuit of material to the pursuit of spirit, social economy gradually steps into the intelligent era. The development of the Internet technology and its application in industry is a new information technology revolution and industrial revolution of the main force that provides a solid technical support for the development of intelligent world [1].

The smart home system with the Internet of things technology is based on real-time remote sensing information environment to expand the traditional control mode to the Internet and mobile terminal. The Internet and Mobile terminal will interact with the traditional entity control and together they form a virtual network control, so as to constitute a set of access to the information, intelligent control, security, remote monitoring functions as one of the core intelligent system. From the systematic point of view, the smart home platform could be separated into the perception layer, network layer and application layer, respectively. Perception layer includes all kinds of the ZigBee carried wireless communication module of the household appliances, lighting equipment, security equipment and so on to achieve the comprehensive perception of the family environment. The perception layer also contains intelligent home gateway that realizes the perception layer and network layer data interaction. Network layer includes a database server and a website to the server. The database server is used to carry out the data interaction with the data gateway of the family by accessing the website to get the information. Application layer includes a web browser based on terminal equipment and the user can utilize a web browser to access site management and control of the whole smart home system [2].

The traditional smart home systems are made of single communication pattern with similar data transfer protocol that will restrict the expansibility. To enhance the performance of the system, we conduct research on the middleware technology and the cross-platform communication that assisted in the architecture of the smart home platform. Middleware was put forward by IBM in 1994 and quickly developed into the construction of the key technology of distributed information system and 
operating system [2]. Middleware is the main trend to the extension of the Internet. It has got rapid development in the world, but with the continuous development of network terminal hardware platform and system, the use of network protocol is still a challenging work. To deal with this issue, we introduce cross-platform communication technique into system. In order to achieve comprehensive monitoring data acquisition and real-time transmission to meet the real-time analysis and processing of large production data, we adopt the isomerism distributed cross-platform data transmission system. This system can refer to the different locations in the sensor network state of a variety of the sensor devices environmental perception, and the data real-time transmission with analysis and processing to provide the users with advanced control priority [2]. The rest of the paper is organized as the follows. (1) We review the state-of-the-art research results on smart home to serve as our background. (2) The basic components of the classic smart home system are introduced with sample systematic illustration. (3) Our proposed middleware technology and the cross-platform communication based modification approaches are discussed. (4) The prospect and future research orientations are finalized.

\section{Literature Review}

Mert's group conducted review on collaborative smart home technologies for the senior independent living [3]. They pointed out that aged population is a serious problem in many developed countries including Canada, where $12.5 \%$ of the overall population accounted for $42.7 \%$ of the total health expenditure. Information technology, like the smart home system can create an efficient healthcare delivery system while realizing elderly's wish of "aging at home”. One project in academia is the Alarm.NET that has been developed by University of Virginia. Alarm.NET includes wireless sensor devices worn by residents to detect activity pattern classification and physiological sensing; tailored network that can provide personal notifications (e.g., alerts to take medicine); emplaced sensors in the living spaces to sense the environmental quality. The future improvements have been identified, including affordability with low cost technologies as well as information security and privacy issues, etc. Perumal proposed the interoperability framework for smart home systems in [4]. They indicated that the smart home system contains different devices, like home automation, cameras, fire alarms, energy management appliances. Researchers proposed a new intelligent interoperability framework for smart home systems executions to realize the message exchange between different devices and federated decision making without external participation. This framework is based on SOAP and web services technologies. The components of the interoperability framework include the application interface, the service stub and database module. Researchers implemented this framework in the home server that uses small form factor system. The result showed that this framework is an ideal choice for managing diversified devices and appliances in home environment. In [5], ZigBee based voice control system for the smart home is proposed. The paper is about the overall design of the wireless home automation system that can respond to voice commands and control the on/off status of the electrical devices, such as lamps, fans, television etc. New technologies such as GSM/GPRS networks, wireless sensor networks and Bluetooth are used in the corresponding system. More related research results can be referred from literatures such as [6-8].

\section{The Proposed Methodology}

The Classic Smart Home System Architecture. Smart home as a part of the Internet of things and people's life is the most direct way people feel the Internet of things. Through the smart home system, people will feel the convenience, rapidness, and intelligence brought by the Internet revolution. According to the review, the architecture of the classic smart home platform can be summarized as follows.

o Information processing module. Its function is mainly to collect all the working status of home appliance in the family and the service request, for a variety of data real-time processing, and send the results to the function driver module. 
o Function driver module. Due to the various electrical appliances manufacturers, products are quite different in the function and implementation, and so will have to pass the function driver module information processing module instruction translation into the electrical friendly level signal, electrical appliances and various state information is converted into binary information that the processing module can understand.

o Communication module. According to the characteristics of family network, communication module uses the existing wiring, or uses wireless transmission, etc. For different information home appliances, the transmission bandwidth requirements are different, so the actual mixed network communication module is often achieved through a variety of ways.

o Outside information interface module. The module can be a gateway to the outside world, a family in all home appliance inside the family, on the basis of the information sharing, further implement the resources sharing based on the Internet.

Network technology in general can be divided into two kinds: cable network and wireless network technology. At present, the cable network technique is applied to telephone lines, power lines and the Ethernet, etc. However, all of the cable network has varying degrees of wiring trial, including poor scalability, shortcomings and so on that will affect the beauty of the household. Emerging wireless networking technology in the field of smart home application has unique advantages, such as easy to install and maintain, easy to transfer and extend, especially suitable for the household of flexible application scenario. These advantages make the wireless networking technology gradually replace the cable network technology and have become the mainstream in smart home system peripheral network networking technology. Correspondingly, we show the sample architecture in the figure one.

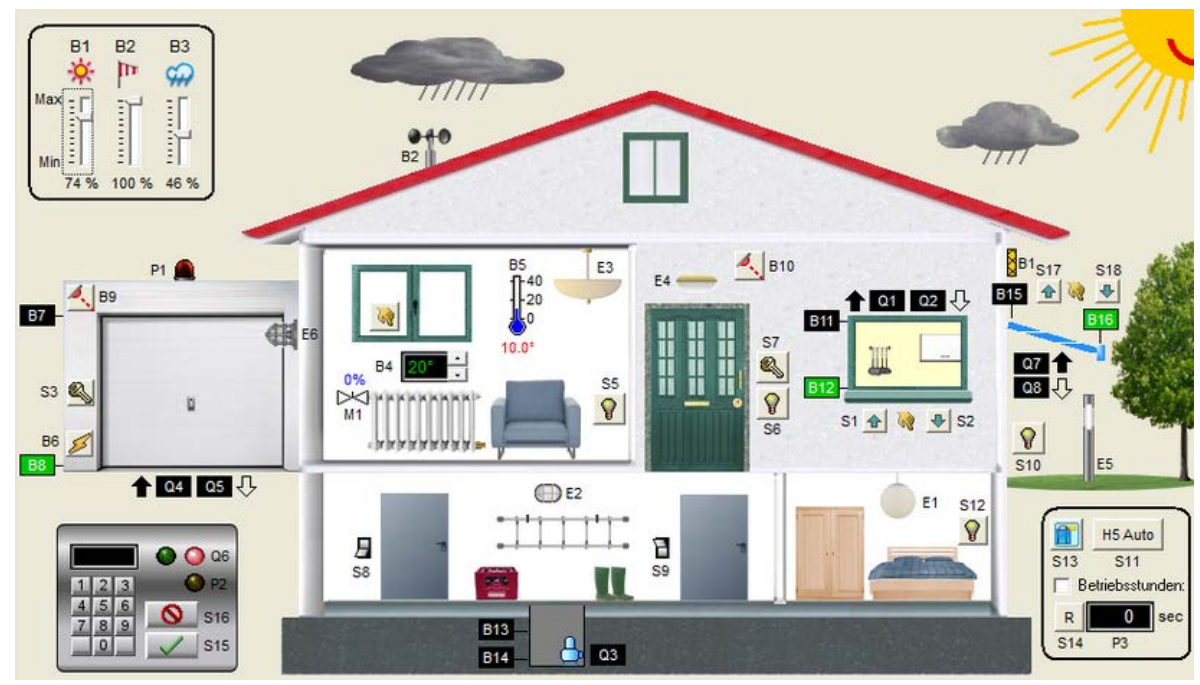

Fig. 1The Sample Architecture of the Modern Smart Home Systems

The Middleware Technology and the Applications. Middleware technology is along with the network and developed a kind of object oriented technology. Its role is mainly used for shielding the differences of network hardware platforms, operating systems and network protocol heterogeneity, enables application software to run more smoothly in the different platforms. At the same time the middleware plays a big role in load balancing, connection management and scheduling that increase the performance of enterprise applications increase greatly to meet the needs of the key business. In our research, we will integrate the gateway page adaptive middleware and database middleware to form our enhanced middleware architecture.

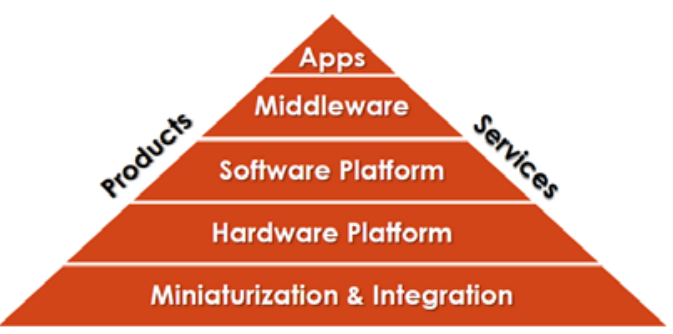

Fig. 2The Technical Grade of Middleware Technology 
Database middleware is the upper control interface and the lower bridge of communication and exchanging data between intelligent household devices. It is designed to provide different kinds of upper interface and the lower equipment the generic driver. Gateway and database middleware that come from the different manufacturers, different communication mode of multiple device nodes can be connected to this topic on the design of the system, and carry on the unified monitoring. Users can monitor the state of the equipment through the web page, or change the data in the database to realize the control of the node. Database middleware's main tasks include: the gateway to upload the data collection to the state of each node according to the number of database; Target state of the user sends to the database by comparing the current state and the target state query and corresponding control instructions; The instruction and node number is sent to the gateway, the gateway according to the number sent to the corresponding equipment so as to complete the user control of the node.

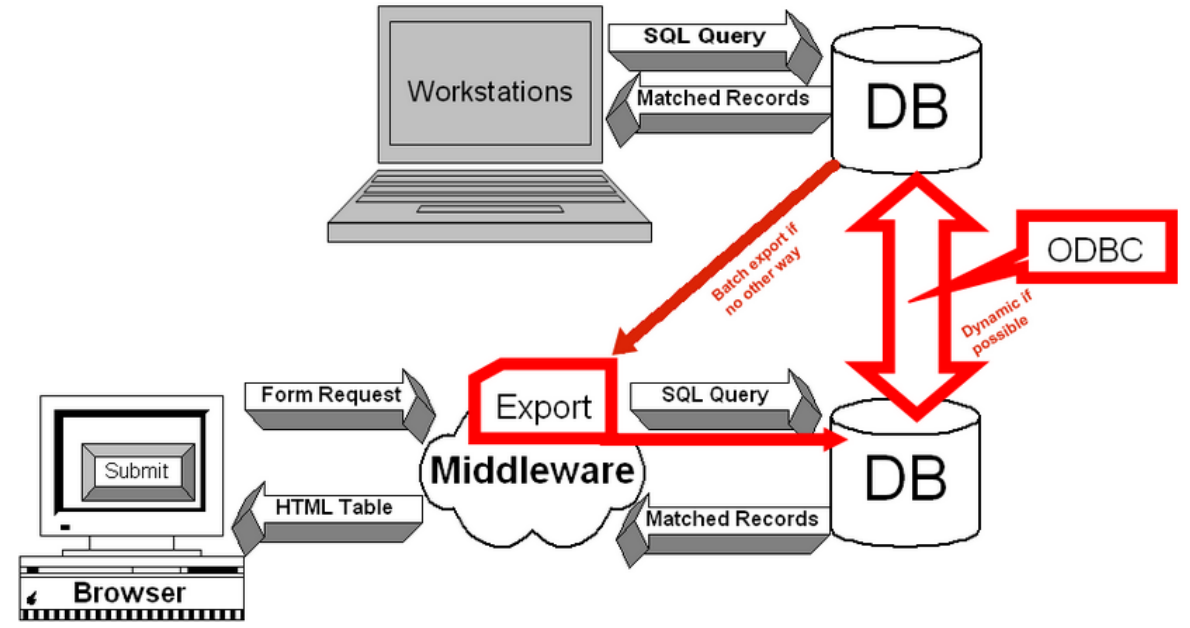

Fig. 3The Demonstration of the Database Middleware

As show in the figure 3, the work flow of the database middleware is illustrated. We use the Mysql database running under the Windows environment. Mysql is the free software developed by the Swiss company MysqlLab which is a real quick, multi-user, multi-threaded SQL database server. The basic functionalities of the middleware are divided into the listed parts. (1) Upload data. Gateway to the serial number of device nodes to collect information and distribution, the WiFi sent to the server, PC communication module and gateway to establish a socket communication, communication module receives the data after the data stored in the database. (2) Give an instruction. Communication module from the instructions in the table query according to the data format in the table format, then add the gateway pre-allocated device node number is sent to the gateway to control the appropriate nodes.

Smart home gateway page within the adaptive system is the central idea that is from existing web pages by tinkering with its data processing finished seamless transition of the page. System to achieve the function as a data flow processing line according to the data flow and processing procedure can include five modules: data acquisition module, data standard module, parsing extracting module, the mapping transformation module, rendering beautification module. For the first time to access the web pages, data acquisition module starts to grab the equipment for web content into data preprocessing, formal module will be normal after the original XML stream into analytic extraction module; Parsing through the analysis of the original XML stream extraction module and get clear information page structure and content to form to parse the core XML stream into the mapping transformation module; Mapping converter for layout templates in the warehouse and the data using XSLT calls the template selection and transformation and ultimately form a unified XML stream, as system also beautify the renderer to add page to make the purpose of the web page seamless transition appears on the screen.

Accordingly, the benefits of adopting gateway page adaptive middleware can be organized as the follows. (1) Scalability. Universal data access and template can greatly continuation and extension of existing programs and services in the application of various new computing scenes. (2) Univer- 
sality. Adaptive system generality is in support of numerous web pages and support many home appliances two aspects, even if the network operators do not have peculiar to open web pages in intelligent home appliance, the gateway middleware can also through the web page to adaptive transform to achieve seamless browsing. (3) Intelligentialize. Through the website of the data information processing and display according to different styles of different terminal equipment layout mapping template and the rendering template design effect of the display of the page.

The Cross-Platform Communication. Cross-platform middle structure research becomes a hot topic in the field of mobile Internet, smartphone applications and the cross-platform framework of innovation is beyond abstract pattern of cross-platform desktop framework of the 1990s. At present, the mobile middleware solutions on the mobile terminal cross-platform development problem solving thinking is divided into the following aspects.

o Introducing the idea of adaptive strategy, under what circumstances specified in the policy configuration file using what kind of adaptation in order to adapt to process control software.

o Using aspect-oriented programming technology to improve reflective component technology, as will be scattered or distribution on the various components related to an application module function through aspect-oriented language refactoring, so can reduce the application system of each component with the relationship between complexity reduce loading error probability.

o Using the reflective component technology, development artifacts, internal implementation details of when the application needs to change, system can be a number of ways from the interface to access and manipulate the runtime components of internal state and behavior. The techniques contain the ACT and Correlate [9].

We will move the system platform of the local development mode combined with the base web development mode and the terminal between the application and server added to design a middleware structure model and used for the upper each mobile terminal system platform of each single different attributes. Mainly in the middleware structure model using web service framework technology, web services for terminal heterogeneous platform resource synergy provides a unified method effectively. For terminal platform, web services is the business logic of the corresponding module encapsulation for interface, this interface with the WSDL description, blocking the terminal platform development and the differences of language and development environment.

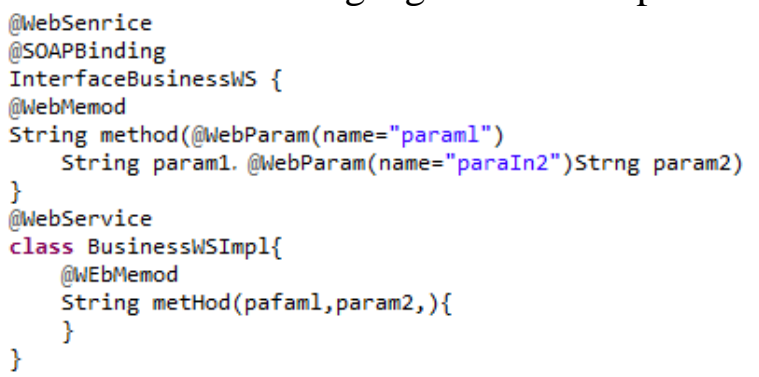

Fig. 4The Sample Code Block of the Web Service Assisted Communication Script

In the system application, the three layer architecture business logic on server side implementation and the main show is blocking layer application middleware structure model platform differences to concentrate the business logic in a layer with system integration and data interaction. In the system application, the process of collaboration between each layer can be summarized as follows. (1) The business logic layer. The business logic layer is a web-based application development server to realize the business logic of the whole system function. Tomcat as the server and database connection use the JDBC directly connected or use a third party framework hibernate, as long as the parameter setting to specify the database configuration files. System by asking a structure model to rebuild the business layer, encapsulating business module to the corresponding interface through a web service published to the registry and calls for the mobile terminal platform accordingly module. (2) Abstract based on the middleware structure unified platform service access interface. The middleware structure model provides an integrated platform of existing common method, equivalent to an intermediate service access interface that can be adapted to conform to the rules of the target sys- 
tem for platform SDK interface with integration of the mobile platform and for future possible new system platform reserved channel access that is easy to extend. (3) The transition of middleware structure model to the target platform. In the platform to interact with each mobile system, must eventually by the target system of the SDK to corresponding method for processing that is the introduction of different third party class library to parse the XML file. Due to the differences between various mobile system platforms, to realize the transformation of interface is not able to use a unified approach to global transformation, so for each target platform needs to operate alone.

\section{Conclusion and Summary}

In this paper, we conduct research and deployment on smart home system based on the middleware technology and cross-platform communication. The development of the Internet technology and its application in the industry is a new information technology revolution and industrial revolution of the main force for the development of intelligent world that provides a solid technical support. Under this background, we integrate the middleware techniques and cross-platform communication to optimize traditional smart home. Middleware is mainly used for shielding the differences of network hardware platforms, operating systems and network protocol heterogeneity to enable application software to run more smoothly in the different platforms. Cross-platform communication could satisfy the basic need of data interaction between different platforms. In the future, we will combine hardware devices to implement the proposed systematic framework and enhance the performance.

\section{References}

[1] Hargreaves, Tom, et al. "Smart homes, control and energy management: how do smart home technologies influence control over energy use and domestic life?." (2015).

[2] Arora, Nand Nandan, et al. "A novel smart meter for better control over devices including electric vehicles and to enable smart use of power in smart home." Transportation Electrification Conference (ITEC), 2015 IEEE International. IEEE, 2015.

[3] Bal, Mert, et al. "Collaborative smart home technologies for senior independent living: a review." Proceedings of the 2011 15th International Conference on Computer Supported Cooperative Work in Design (CSCWD). 2011.

[4] Perumal, Thinagaran, Abdul Rahman Ramli, and Chui Yew Leong. "Interoperability framework for smart home systems." Consumer Electronics, IEEE Transactions on 57.4 (2011): 16071611.

[5] Krishna, Y. Bala, and S. Nagendram. "Zigbee based voice control system for smart home." International Journal on Computer Technology and Applications 3.1 (2012): 163-168.

[6] Hu, Qinran, Fangxing Li, and Chien-fei Chen. "A Smart Home Test Bed for Undergraduate Education to Bridge the Curriculum Gap From Traditional Power Systems to Modernized Smart Grids." Education, IEEE Transactions on 58.1 (2015): 32-38.

[7] Ye, Juan, Graeme Stevenson, and Simon Dobson. "Fault detection for binary sensors in smart home environments." IEEE International Conference on Pervasive Computing and Communications (PerCom 2015). IEEE Computer Society, 2015.

[8] Nagar, Seema, et al. "A Framework for Evaluating the Costs and Benefits of Instrumentation in Smart Home Systems." Proceedings of the 2015 ACM Sixth International Conference on Future Energy Systems. ACM, 2015.

[9] Chaczko, Zenon, et al. "Haptic Middleware Based Software Architecture for Smart Learning." Computer Aided System Engineering (APCASE), 2015 Asia-Pacific Conference on. IEEE, 2015. 